\title{
Primary Steps for Handling Childhood Obesity
}

\author{
Jennifer Jasmin Khan, MBBS \\ University of South Carolina, Arnold School of Public Health
}

\section{Introduction}

Childhood obesity represents an important public health concern in $21^{\text {st }}$ century. The condition has reached epidemic proportions in developed countries and has significant rates in some developing countries. Its prevalence is said to have tripled over the past 30 years, with an estimated number of overweight children globally at about 42 million. Of these numbers, about 35 million live in developing countries such as Nepal, Pakistan, India and Bangladesh (WHO, 2013 1). In Europe, Scandinavian and Mediterranean countries record in increased childhood obesity rates. Iran is reported to be among the top seven countries with the highest prevalence of childhood obesity, and in Saudi Arabia one out of every six children between ages 6 and 18 is obese (Dehghan, Akhtar-Danesh and Merchant, 2005). In the United States, childhood obesity has doubled among young children and had tripled among adolescents since 1980. The 1999-2002 National Health and Nutrition Examination Survey (NHANES) revealed that 16 percent of children between ages 6 and 19 are overweight. The heaviest children in the survey were significantly heavier than what was recorded in previous surveys. Obesity rates were also higher for some minority youth populations. For both developed and developing countries, a higher proportion of obesity was recorded among girls, rather than boys (Dehghan, Akhtar-Danesh and Merchant, 2005). Obesity in childhood and adolescence carry the risk of the development of orthopedic problems, respiratory conditions, hypertension, depression, as well as Type 2 diabetes while still a youth. Demonstrating the disproportionate obesity trends among minority youths, Type 2 diabetes has increased significantly in African American, American Indian and Hispanic/Latino children and youth. Furthermore, there is a $70 \%$ chance that overweight adolescents will become obese or overweight adults. Obesity in adulthood in turn increases the risk for asthma, arthritis, high blood pressure, diabetes, high cholesterol and other adverse health conditions. Overweight and obesity are largely preventable. Their prevention eliminates related health adversities and enables a better quality of life for young people while reducing burdens on the health care system (Bishop et al, 2005; Dehghan, Akhtar-Danesh and Merchant, 2005). Prevention of childhood obesity is therefore a high priority for public health. This paper reviews primary interventions for handling childhood obesity, looking at its features, causes and intervention methods.

\section{Clinical Features of Childhood Obesity}

The physiological changes that happen with age mean that the indices for defining obesity and overweight change with time. However, a common clinical determinant is an excess of body fat (Freedman et al., 2007; Lohman, 1991; WHO 2013 2). Overweight refers to an excess body weight from muscle, bone, water or for a combination of these for a given height. Obesity is described as having an excess of body fat (Centers for Disease Control and Prevention (CDC), 2013). A consensus on the cutoff point for excess fat for overweight and obesity does not exist. One study classified children ages 5 to 18 as obese if their body fat percentage was $25 \%$, and $30 \%$ for males and females respectively based on a skin fold thickness of 3320 . The WHO has a growth reference for children 5 to 19 years of age that is based on height, weight and body mass index (BMI) (WHO, 2013). The Center for Disease Control and Prevention further define "overweight" as being "at or above the 95th percentile of BMI for age", and "at risk for overweight" as being "between 85th to 95th percentile of BMI for age". European researchers categorize overweight as "at or above 85th percentile and obesity is categorized as "at or above 95th percentile of BMI".

There are several methods to measure body fat. Research techniques include magnetic resonance imaging (MRI), multi-frequency bioelectrical impedance analysis (BIA) and underwater weighing (densitometry). Clinical techniques include body mass index (BMI), skinfold thickness and waist circumference. While clinical methods are not as accurate as research methods, but they are effective for risk identification. BMI is more efficient for adults than for children because of physiological changes that occur as they grow. Also, BMI does not distinguish between fat-free mass (muscle and bone) and fat and this can impact on obesity assessment for muscular children. Bioelectrical impedance analysis (BIA) is used in populationbased studies as well as in clinical settings. BIA predicts fat mass or percentage of body fat (\%BF), fat-free mass (FFM and total body water (TBW) among children. Waist circumference is also effective for children as it identifies central obesity (Dehghan, Akhtar-Danesh and Merchant, 2005; Freedman, Wang, Ogden et al., 2007). 


\section{Causes of Childhood Obesity}

While overweight and obesity are outcomes of caloric imbalance, behavioral, environmental and genetic factors also impact on these conditions (CDC, 2013). The agents and factors that predispose children to obesity occur from infancy and all through youth.

Breastfeeding: The practice of breast feeding can protect children against overweight and obesity. In the U.S., an estimated $75 \%$ of mothers adopt breastfeeding but by the end of six months, only $13 \%$ of babies receive exclusive breastfeeding (Owen, Martin and Whincup, 2005; Rudolf M, 2011).

Media Use: Children between ages 8 and 18 spend an estimated 7.5 hours on entertainment media each day; these include video games, TV, cell phones, computers and movies. About 4.5 hours are spent on TV alone. These activities impact on childhood obesity as they reduce the time spent on physical activities; advertisements influence children to make food choices that are not healthy, and there is increased energy intake from eating while watching TV (Robinson, 2001; Zimmerman\& Bell, 2010).

Negative Media Advertising: Children's food and snack choices are being influenced by media campaigns. There are more advertisements for foods that have high calorie contents; sugar, fat and salt, as against foods that are healthy and nutritionally balanced.

Insufficient Physical Activity: Studies show a strong correlation between weight gain and low levels of physical activity. Decline in physical activity is believed to be responsible for global increase in obesity rates (Dehghan, Akhtar-Danesh and Merchant, 2005). It is recommended that children be trained to be physically active, especially through implementing active physical education programs in schools. Daily, Quality physical education carried out in schools can help children meet the requirements the prevention of childhood obesity. An hour of aerobic physical activity daily can reduce childhood obesity rates by half (Bishop et al, 2005; Fulton et al., 2004; WHO 2013 1).This factor can in turn be linked to an environmental issue whereby children have no safe or appealing place to play. Access to recreation centers and parks may be difficult for some families; safe biking and walking routes may not exist, and transportation to recreational facilities might be a problem for some families.

Nutritional Status and Access to Foods: The socio-economic status of families affects their nutritional status and access to food. Some families have reduced access to supermarkets and grocery stores that sell foods such as vegetables and fruits. This occurs mostly in lower-income and minority-dominated neighborhoods. At the same time, families living in areas where there are numerous retailers (such as fast food restaurants and convenience stores)that sell less healthy food, tend to consume more of such type of foods. Another aspect of nutritional intake and its consequences for children's weight is the high availability of sugary drinks and energydense foods. Sugary drinks are an important factor in high calorie intake for children. Students have easy access to less healthy foods and sugar drinks at school from school cafes, vending machines and activities such as parties, fundraising and sporting events (Dehghan, Akhtar-Danesh and Merchant, 2005; Sondike, Copperman and Jacobson, 2003).

\section{Primary Intervention}

Metabolic programming, developmental and environmental factors play a role in weight regulation. Of the three, environmental factors can be modified from infancy and childhood. Prevention and treatment of childhood obesity primarily focuses on the modification of behaviors that correlate with obesity and weight gain. At the same time, long-term randomized trials to determine effective treatment or prevention techniques for obesity are few; techniques applied in clinical trials may not be practical for clinical settings. Recommended approaches are based on observations of behaviors that are associated with obesity, clinical experience and short-term trials (Barlow, 2007; Skelton, 2013). Nonetheless, a number of approaches are recommended by expert consensus, based on clinical experience, inferences drawn from observing obesity-associated behaviors, and short-term evidence-based trials. General guidelines adopted for obesity interventions include tracking BMI changes over time, routine counseling and routine assessment for risk factors. Routine interventions include education, family centered interventions as against a focus on the children alone, encouraging long term behavioral changes and staged weight management (Barlow, 2007; Skelton, 2013; Whitlock, O'Connor, Williams, 2010). The staged approach to weight management is based on the child's BMI percentile, age and prior weight management history. Interventions are divided into stages: prevention plus - stage 1, structured weight management - stage 2, multidisciplinary evaluation - stage 3 , and tertiary care -4 (Ross et al, 2010; Skelton, 2013, Whitlock, O'Connor, Williams, 2010).

\section{Weight Managements Strategies}

A number of steps are adopted for weight management; these mostly address the environmental dimensions that impact on the prevalence of obesity. Weight management strategies fall under four broad groups: behavioral strategies, motivational interviewing, family centered communication and scare tactics (Hill, Chapman and Donovan, 1998; Suarez and Mullins, 2008; Whitlock, O'Connor, Williams, 2010). 
Behavioral Strategies

Established counseling techniques that are used for treatment in pediatric obesity treatment adopt a behavioral change model that includes self-monitoring, goal setting, stimulus control and positive reinforcement (Ross et al, 2010; Suarez and Mullins, 2008; Whitlock, O'Connor, Williams, 2010).

Self-monitoring of risk behaviors can include food and activity logs. The patient and their family are able to determine the behaviors responsible for weight gain through this process. The process is overseen by a physician. Other contributors to obesity such as level of hunger and meal environments can be detected in the process.

Goal-setting is a widely used technique for prompting behavior change; the set goals are health related rather than weight related specifically. The process is efficient where the goals are maintainable and realistic. The popular "SMART" criteria can be applied. Goals should be specific, measurable, attainable, realistic, and time bound.

Stimulus control is aimed at reducing the environmental cues that facilitate unhealthy behaviors. At the same time, an effort is made to create an environment that promotes health. Removing televisions from the bedroom or unhealthy foods from the home can help reduce negative behaviors; increasing access to vegetables and fruits can make for enhanced health outcomes.

Positive reinforcement for target behaviors can encourage positive behaviors. This can include praise and rewards agreed on by the child and their parent, with inputs from the health care provider. Rewards should be privileges and activities that have positive health impacts rather than food or monetary incentives. This is related to contracting for rewards. Contracting is an agreement to mark the achievement of specific goals with a reward. This provides structure and focus to goal-setting for the children (Ross et al, 2010; Suarez and Mullins, 2008; Whitlock, O'Connor, Williams, 2010).

Motivational Interviewing

Motivational interviewing is increasingly being used for obesity treatment. This technique focuses on changing a patient's ambivalence to change using their values as a vehicle. Reflective listening is adopted by the physician in order to encourage the patient to identify their reasons for needing behavior change and solutions. Motivational interviewing adopts an encouraging, empathic and nonjudgmental tone. Numerical analogs or interviewing can be used to assess the patient's readiness to change. This assessment helps the patient and the clinician identify ambivalence as a key step in behavior change. Few specific goals that are related to overall health and weight management should be selected for focus, rather than goals targeting weight loss specifically (Suarez and Mullins, 2008; Whitlock, O'Connor, Williams, 2010).

Scare Tactics

Scare tactics are conversations that emphasize health consequences. The clinician has the responsibility of educating the patient and their family on the risks associated with overweight and obesity. This can include providing accurate information on a child's weight based on a growth chart, and describing the health implications of excess body fat. The use of this tactic however is not recommended. While they might attract some immediate attention, they do not make for long term changes as people do not necessarily think probabilistically. Also, response to risk-based thinking is not consistent. It is more efficient to focus on less dire outcomes such as: reduced athletic ability, reduced mobility, adult obesity, or any immediate health experiences of patients and their families (Hill, Chapman and Donovan, 1998).

Family-centered communication

Effective techniques in behavior change are patient centered or family centered; behavior change efforts should be collaborative and not prescriptive. Families should be engaged in a conversation to select goals for behavior change. Children can be involved in decision-making where they are old enough to participate. This ensures that the patient and their family can implement the intervention plan with the confidence that goals are achievable, and also be committed to the process. These factors enhance the chances of success (Ross et al, 2010; Suarez and Mullins, 2008).

\section{Prevention Strategies}

A good understanding of the causes of obesity provides opportunities to design interventions that are beneficial and effective to individuals and communities. Prevention is agreed to be the key strategy for controlling obesity. This includes primary and secondary prevention strategies. Evidence suggests that achieving modifications on these factors impact on obesity prevalence.

Physical Activity

A recent study revealed that a one-hour increase in physical education and related activity resulted to a $1.8 \%$ BMI reduction among at-risk and overweight girls in first grade (Dehghan, Akhtar-Danesh and Merchant, 2005). Vigorous physical activity should be encouraged for one or more hours a day. Television and screen time should be limited for children; this can include strategies such as less than two hours daily limits and no screen time for children who are below two years of age(Bishop et al, 2005; Fulton et al., 2004; WHO 2013 1). 
Maternal Behavior

Maternal weight before conception and weight gain after conception should be controlled by healthy diets and activity, as this can contribute to baby's risk for obesity. Breastfeeding has a weak effect in preventing obesity; however exclusive breastfeeding for up to 6 months is beneficial (Owen, Martin and Whincup, 2005; Rudolf M, 2011; Whitaker et al. et al, 1997).

Dietary goals

Nutritional management of obesity is important and effective. Dietary goals can include limiting the consumption of sugar and beverages that are sweetened with sugar. Limiting eating at fast-food restaurants and increasing vegetable and fruit intake can be very helpful. Portion sizes can also be limited. Reduced glycemic load diet is being used in the treatment and management of adolescent obesity (Dehghan, Akhtar-Danesh and Merchant, 2005; Ebbeling et al., 2003)

\section{Psychosocial factors}

Factors such as encouraging family meals and establishing healthy feeding pattern early in childhood can help reduce the prevalence of childhood obesity. Feeding patterns should not be too restrictive or overly permissive. Healthy regimens such as fruits and vegetables should be encouraged early in life.

\section{Conclusion}

Excess calorie intake that is above the daily energy expenditure leads to weight gain, and subsequently, to childhood obesity. While genetic factors can impact on the process, physical activity and diet are two main variables in the development of obesity. These are in turn largely determined by socio-economic status, physical activity, advertising influences and to some degree by race and ethnicity. The causes of obesity are therefore multi-factorial. Prevention is the key step to handling childhood obesity, and is based on a good knowledge of the causes of obesity. Primary prevention techniques include counseling, dietary changes, behavioral change and increased physical activity. Healthy maternal behaviors such as weight control during pregnancy through healthy eating and safe exercise is important towards reducing risks for the child. Breastfeeding for up to 12 months is optimal for children and can play a role in preventing obesity. Primary prevention and health management strategies can be used for direct prevention as well as managing at-risk situations. For children who are already obese or overweight, primary prevention is no longer an option. In such cases, structured weight management interventions are necessary. However, to most effectively battle this childhood obesity epidemic, the prevention strategies are not enough only. We need both clinical research and changes to public policy and government regulations to face this epidemic as well.

\section{Works Cited}

[1]. Barlow SE, 2007. Expert Committee. Expert committee recommendations regarding the prevention, assessment, and treatment of child and adolescent overweight and obesity: summary report. Pediatrics; 120 Suppl 4:S164.

[2]. Bishop J, Middendorf R, Babin T and Tilson W 2013. Available at < http://aspe.hhs.gov/health/reports/child_obesity/index.cfm\#_ftn4> [Accessed November 27, 2013]

[3]. Centers for Disease Control and Prevention (CDC) 2013 .Childhood Obesity Facts. Available at $<$ http://www.cdc.gov/HealthyYouth/obesity/facts.htm> [Accessed November 27, 2013]

[4]. Dehghan M, Akhtar-Danesh N and Merchant AT 2005. Childhood obesity, prevalence and prevention. Nutrition Journal, 4:24 doi:10.1186/1475-2891-4-24

[5]. Ebbeling CB, Leidig MM, Sinclair KB, et al. 2003. A reduced-glycemic load diet in the treatment of adolescent obesity. Arch Pediatr Adolesc Med; 157:773.

[6]. Freedman DS, Wang J, Ogden CL, et al. 2007. The prediction of body fatness by BMI and skinfold thickness among children and adolescents. Ann Hum Biol; 34:183.

[7]. Fulton JE, Garg M, Galuska DA, et al. 2004. Public health and clinical recommendations for physical activity and physical fitness: special focus on overweight youth. Sports Med; 34:581.

[8]. Hill D, Chapman S, Donovan R 1998. The return of scare tactics. Tob Control; 7:5.

[9]. Lohman TG. Anthropometric assessment of fat-free body mass. Wile-Liss, 1991.

[10]. Owen CG, Martin RM, Whincup PH et al. 2005. Effect of infant feeding on the risk of obesity across the life course: a quantitative review of published evidence. Pediatrics; 115:1367- 1377.

[11]. Robinson TN 2001. Television viewing and childhood obesity. Pediatr Clin North Am;48(4):1017-25.

[12]. Ross MM, Kolbash S, Cohen GM, Skelton JA 2010. Multidisciplinary treatment of pediatric obesity: nutrition evaluation and management. Nutr Clin Pract; 25:327.

[13]. Rudolf M 2011. Predicting babies' risk of obesity. Arch Dis Child; 96:995

[14]. Skelton JA 2013. Management of childhood obesity in the primary care setting. Available at $<$ http://www.uptodate.com/contents/management-of-childhood-obesity-in-the-primary-care- setting $>$ [Accessed November 27, 2013]

[15]. Sondike SB, Copperman N, Jacobson MS. Effects of a low-carbohydrate diet on weight loss and cardiovascular risk factor in overweight adolescents. J Pediatr 2003; 142:253

[16]. Suarez M, Mullins S. Motivational interviewing and pediatric health behavior interventions. J Dev Behav Pediatr 2008; $29: 417$.

[17]. Whitaker RC, Wright JA, Pepe MS, et al. 1997 Predicting obesity in young adulthood from childhood and parental obesity. $N$ Engl J Med; 337:869. 
[18]. World Health Organization (WHO) 2013. Childhood overweight and obesity. Available at $<$ http://www.who.int/dietphysicalactivity/childhood/en/> [Accessed November 27, 2013]

[19]. Zimmerman FJ, Bell JF 2010. Associations of television content type and obesity in children. Am J Public Health;100(2):334 - 40.

[20]. Center for Disease Control and Prevention. <http://www.cdc.gov/healthyyouth/obesity/facts.htm>

[21]. Heidi M. Blanck, Sonia A. Kim (September 2012). Creating Supportive Nutrition environments for Population Health Impact and health equity. American Journal of Preventive Medicine. Volume 43, Issue 3, Supplement 2.

[22]. Y. Claire Wang, Amber Hsiao, C. Tracy Orleans, Steven L. Gortmaker (2012). The Caloric Calculator- Average Caloric Impact of Childhood Obesity Intervention. American Journal of Preventive Medicine. Volume 45, Issue 2.

[23]. Jay e. Maddock PhD (September 2012). Addressing Physical Activity, Obesity and Wellness in Schools. American Journal of Preventive Medicine. Volume 43, issue 3.

[24]. Whitaker RC, Wright JA, Pepe MS, Seidel KD, Dietz WH: Predicting obesity in young adulthood from childhood and parental obesity. New England Journal of Medicine 1997, 337:869-873.

[25]. Deurenberg P, Kusters CS, Smit HE: Assessment of body composition by bioelectrical impedance in children and young adults is strongly age-dependent. Eur J Clin Nutr 1990, 44:261-268.

[26]. Lazarus R, Baur L, Webb K, Blyth F: Body mass index in screening for adiposity in children and adolescents: systematic evaluation using receiver operating characteristic curves. Am J Clin Nutr 1996, 63:500-506.

[27]. Stevens J: Obesity, fat patterning and cardiovascular risk. Adv Exp Med Biol 1995, 369:21-27 